\title{
Lombalgia Gestacional: uma Revisão Integrativa sobre Hidroterapia, Reeducação Postural Global e Pilates
}

\author{
Gestational Low Back Pain: an Integrative Review on Hydrotherapy, Global Postural \\ Rehabilitation and Pilates
}

\author{
Flávia Borges Domingues Roma; ; Denise Campos-Pozzi*a

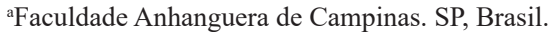 \\ *E-mail: denise.campos@gmail.com
}

\begin{abstract}
Resumo
A gestação compreende um período de várias mudanças anatômicas e fisiológicas no corpo da gestante. Um problema de grande relevância nessa fase é a lombalgia gestacional, que acarreta dor na região lombar e compromete a qualidade de vida da mulher grávida. O objetivo do trabalho foi verificar, através de revisão de literatura, os efeitos da hidroterapia, reeducação postural global (RPG) e Pilates no tratamento da dor lombar durante a gestação. Foram incluídos estudos clínicos de gestantes que estavam no segundo ou terceiro trimestre de gestação, cuja dor lombar surgiu na gestação e não havia associação com dor pélvica, publicados nas bases de dados SciELO, LILACS e Google Acadêmico, disponíveis na íntegra e online, entre os anos de 2010 a 2020. Foram excluídos artigos de revisão de literatura, monografias, que falavam sobre lombalgia decorrente de outras causas e que utilizavam esses tratamentos em homens ou mulheres não gestantes. Foram selecionados 6 artigos, 3 de hidroterapia, 2 de RPG e 1 de Pilates. Verificou-se que os três métodos causaram redução na intensidade da dor lombar gestacional. Além disso, a hidroterapia diminuiu a tensão nos músculos dorsais, aumentou a flexibilidade dos músculos estabilizadores da coluna e melhorou a qualidade de vida das gestantes; e a RPG ocasionou diminuição da limitação funcional e melhora da qualidade de vida. Concluiu-se, a partir desta revisão de literatura, que a hidroterapia, RPG e Pilates proporcionaram melhora significativa da dor lombar durante a gestação, havendo diminuição na intensidade da dor.
\end{abstract}

Palavras-chave: Gravidez. Dor Lombar. Exercícios Terapêutico. Exercícios de AlongamentoMuscular. Exercícios Aquáticos.

\begin{abstract}
Pregnancy represents a period of several anatomical and physiological changes in the pregnant woman's body. A problem of great relevance in this phase is gestational low back pain, which causes pain in the lower back and compromises the quality of life of pregnant women. The objective of the study was to verify, through literature review, the effects of hydrotherapy, global posture reeducation (GPR) and Pilates in the treatment of low back pain during pregnancy. Clinical studies of pregnant women who were in the second or third trimester of pregnancy, whose low back pain appeared during pregnancy and there was no association with pelvic pain, published in the SciELO, LILACS and Google Scholar databases, available in full and online, between 2010 to 2020 were included. The literature review articles and monographs, that were about low back pain due to other reasons and that used these treatments in men or non-pregnant women were excluded. Six articles were selected, three of hydrotherapy, two of GPR and one of Pilates. It was found that the three methods caused reduction in the intensity of gestational lumbar pain. In addition, hydrotherapy decreased the tension in dorsal muscles, increased the flexibility of spine stabilizing muscles and improved the quality of life of pregnant women; and GPR caused decrease in functional limitation and improved quality of life. It was concluded, from this literature review, that hydrotherapy, GPR and Pilates provided significant improvement in low back pain during pregnancy, with a decrease in pain intensity.
\end{abstract}

Keywords: Pregnancy. Low Back Pain. Therapeutic Exercises. Muscle Stretching Exercises. Aquatic Exercises.

\section{Introdução}

Estima-se que 73\% das gestantes apresentam lombalgia (SANTANA; PEREIRA, 2018). A lombalgia caracterizase por um desconforto axial na região lombar inferior, essencialmente musculoesquelético, podendo ocorrer devido a uma combinação de fatores mecânicos, circulatórios, hormonais e psicossociais (BARROS et al., 2018; CUNHA et al., 2018; SANTANA; PEREIRA, 2018).

$\mathrm{O}$ aumento da massa corporal, associado à retenção de líquido e ao aumento dos níveis de vários hormônios, contribuem para aumentar a sobrecarga no sistema musculoesquelético durante a gestação e desencadear a dor lombar (WITTKOPF et al., 2015).
Os sintomas provocados pela lombalgia são responsáveis por várias repercussões negativas na qualidade de vida da gestante, tais como, redução da disposição física, desempenho profissional, vida social e atividades domésticas, ocasionando faltas ao trabalho e redução na produtividade, gerando importante impacto socioeconômico (MADEIRA et al., 2013; SILVA et al., 2017).

Embora a lombalgia seja um sintoma com alta prevalência e tema de estudo em diversos países, esse problema tem sido negligenciado, considerado por muitos profissionais da área da saúde como um desconforto comum na gestação, que não necessita de medidas de prevenção ou alívio (SANTOS et.al., 2018). Dessa forma, é necessária a desconstrução desse pensamento, entre os profissionais de saúde que lidam com 
gestante, e a busca de tratamentos efetivos (SANTOS et al., 2018).

A hidroterapia é baseada nos efeitos dos princípios físicos no corpo em imersão, como empuxo, pressão hidrostática e viscosidade. O corpo imerso sobre efeito do empuxo apresenta menor descarga de peso articular, maior liberdade de movimentação e alívio dos quadros álgicos (SCHEFFER et al., 2018). Além desses benefícios, a hidroterapia ajuda no ganho de força muscular, melhora a respiração, possibilita o alongamento, relaxamento muscular e ameniza as tensões provocadas pelas mudanças posturais na gestante (BARROS et al., 2018).

A Reeducação Postural Global (RPG) é uma atividade que se baseia no alongamento global dos músculos estáticos antigravitacionais, músculos rotadores internos e respiratórios, organizados em cadeias musculares e alongados simultaneamente por aproximadamente 15 a 20 minutos (TERRA et al., 2015). Além de não promover efeitos colaterais, contribui para diminuição das tensões musculares na cadeia posterior, principalmente nos músculos paravertebrais da região lombar (MARCHIORI et al., 2020).

Já o Método Pilates tem se destacado dentre as modalidades terapêuticas de baixa intensidade descritas na literatura, especialmente pela base de seus exercícios, considerados seguros e que enfatizam o trabalho da musculatura estabilizadora da coluna lombar (COTA; METZKER, 2019). Esta técnica requer a realização dos movimentos de maneira harmoniosa, utilizando os princípios básicos de equilíbrio, concentração, precisão e respiração (COTA; METZKER, 2019).

Tendo em vista que a lombalgia gestacional é um sintoma bastante frequente, que acarreta muitas repercussões na vida da gestante, o presente estudo teve por objetivo verificar, através de revisão de literatura, os efeitos da hidroterapia, RPG e Pilates no tratamento da dor lombar durante a gestação. Este trabalho contribui então para divulgação de conhecimentos sobre a lombalgia gestacional, os métodos utilizados para o tratamento, bem como seus efeitos.

\section{Desenvolvimento}

\subsection{Metodologia}

Trata-se de uma revisão integrativa da literatura sobre a utilização da hidroterapia, RPG e Pilates no tratamento da lombalgia gestacional. Foram incluídos estudos clínicos de gestantes que estavam no segundo ou terceiro trimestre de gestação; cuja dor lombar surgiu na gestação e não havia associação com dor pélvica; publicados nas bases de dados SciELO, Llilacs e Google Acadêmico, disponíveis na íntegra e online, entre os anos de 2010 a 2020. Para pesquisa foram utilizados os seguintes descritores e seus sinônimos, em português e inglês de forma isolada ou combinada: gestação, gravidez, lombalgia, dor lombar, reeducação postural global, RPG, Pilates, hidroterapia, exercícios terapêuticos, exercícios de alongamento muscular, exercícios aquáticos. Foram excluídos artigos de revisão de literatura, monografias, que falavam sobre lombalgia decorrente de outras causas e que utilizavam esses métodos de tratamento em homens ou mulheres não gestantes. A Figura 1 mostra o fluxograma da revisão, contendo critérios de busca, quantidade de artigos selecionados e excluídos em cada etapa.

Figura 1 - Fluxograma da revisão: critérios de busca, quantidade de artigos selecionados e excluídos em cada etapa

\section{Descritores utilizados em português e inglês:}

Gravidez, Gestação, lombalgia, dor lombar, Reeducação postural global, RPG, Pilates, Hidroterapia, Exercícios terapêuticos, Exercícios de alongamento muscular, Exercícios aquáticos.

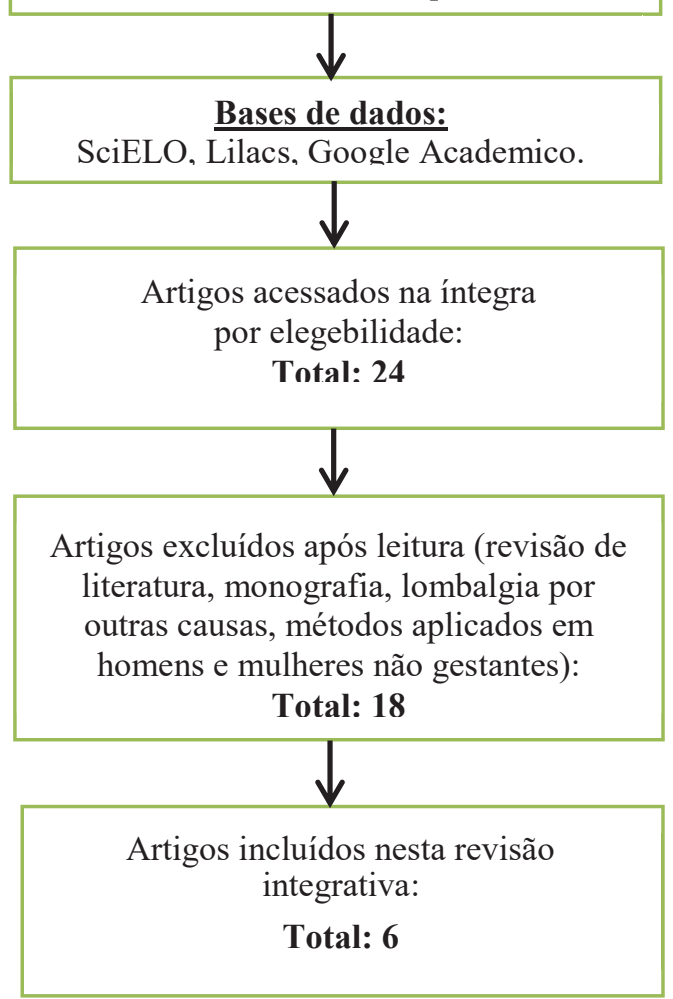

Fonte: Dados da pesquisa.

\subsection{Resultados e Discussão}

Nesta revisão integrativa de literatura foram incluídos seis artigos para análise, os quais atenderam aos critérios estabelecidos e falavam concomitantemente sobre lombalgia gestacional e métodos de tratamento. Dentre os estudos incluídos três abordavam sobre hidroterapia, dois sobre RPG e um sobre Pilates. O Quadro 1 demonstra mostra as características de cada estudo. 
Quadro 1 - Características dos estudos clínicos incluídos nesta revisão

\begin{tabular}{|c|c|c|c|}
\hline Autor/Ano & Amostra & Intervenção/Avaliação & Resultados \\
\hline $\begin{array}{l}\text { Gil et al. } \\
\text { (2011) }\end{array}$ & $\begin{array}{l}\text { n: } 34 \text { gestantes } \\
\text { IG: } 20-25 \text { semanas } \\
\text { Grupo Controle: } 17 \\
\text { Grupo Estudo: } 17\end{array}$ & $\begin{array}{l}\text { RPG: } 8 \text { sessões, } 40 \text { min. } \\
\text { Avaliação: escala visual de dor e } \\
\text { questionário Roland-Morris. }\end{array}$ & $\begin{array}{l}\text { Redução na intensidade da dor, e limitações } \\
\text { funcionais. }\end{array}$ \\
\hline $\begin{array}{l}\text { Sebben, et } \\
\text { al. }(2011)\end{array}$ & $\begin{array}{l}\text { n: } 2 \text { gestantes } \\
\text { IG: } 12-36 \text { semanas }\end{array}$ & $\begin{array}{l}\text { Hidroterapia: } 10 \text { sessões, } 50 \text { min, } 2 x \text { por } \\
\text { semana. } \\
\text { Avaliação: escala visual de dor e exame } \\
\text { físico. }\end{array}$ & $\begin{array}{l}\text { Redução da dor lombar e melhora da } \\
\text { flexibilidade muscular. }\end{array}$ \\
\hline $\begin{array}{l}\text { Souza et al. } \\
(2012)\end{array}$ & $\begin{array}{l}\text { n: } 1 \text { gestante } \\
\text { IG: } 26 \text { semanas }\end{array}$ & $\begin{array}{l}\text { Hidroterapia e solo: } 50 \text { min cada. } \\
\text { Avaliação: eletromiografia da atividade } \\
\text { dos paravertebrais, perimetria de membros } \\
\text { inferiores e escala visual de dor. }\end{array}$ & $\begin{array}{l}\text { Hidroterapia trouxe melhores resultados em } \\
\text { relação ao solo na diminuição de edema dos } \\
\text { membros inferiores, tensão dos músculos } \\
\text { dorsais e intensidade das dores lombares. }\end{array}$ \\
\hline $\begin{array}{l}\text { Justino e } \\
\text { Pereira } \\
(2016)\end{array}$ & $\begin{array}{l}\text { n: } 15 \text { gestantes } \\
\text { IG: } 13 \text { - } 36 \text { semanas } \\
\text { Grupo controle: } 8 \\
\text { Grupo estudo: } 7\end{array}$ & $\begin{array}{l}\text { Pilates: } 10 \text { sessões, } 60 \text { min, } 2 x \text { por semana. } \\
\text { Avaliação: escala visual de dor e avaliação } \\
\text { estabilométrica }\end{array}$ & Melhora da dor lombar. \\
\hline $\begin{array}{l}\text { Barros et } \\
\text { al. }(2018)\end{array}$ & $\begin{array}{l}\text { n: } 1 \text { gestante } \\
\text { IG: } 18 \text { semanas }\end{array}$ & $\begin{array}{l}\text { Hidroterapia: } 9 \text { sessões, } 60 \text { min, } 2 x \text { por } \\
\text { semana. } \\
\text { Avaliação: questionários SF-36, McGill e } \\
\text { escala visual de dor. }\end{array}$ & $\begin{array}{l}\text { Redução da dor lombar e melhora da } \\
\text { qualidade de vida. }\end{array}$ \\
\hline $\begin{array}{l}\text { Marchiori } \\
\text { et al. } \\
(2020)\end{array}$ & $\begin{array}{l}\text { n: } 1 \text { gestante } \\
\text { IG: } 3^{\circ} \text { trimestre }\end{array}$ & $\begin{array}{l}\text { RPG: } 9 \text { sessões, } 60 \text { min. } 2 x \text { por semana. } \\
\text { Avaliação: testes especiais, escala visual de } \\
\text { dor e o questionário Roland Morris. }\end{array}$ & $\begin{array}{l}\text { Redução da dor lombar e melhora da } \\
\text { qualidade de vida. }\end{array}$ \\
\hline
\end{tabular}

n: número de gestantes; IG: idade gestacional, min: minutos.

Fonte: Dados da pesquisa.

De modo geral, os três métodos de tratamento propiciaram melhora significativa da dor lombar durante a gestação. Em todos os estudos houve redução na intensidade da dor. Além disso, cada método trouxe outros efeitos associados. A hidroterapia proporcionou diminuição de tensão nos músculos dorsais, ganho de flexibilidade na musculatura estabilizadora da coluna e melhora da qualidade de vida; e a RPG ocasionou diminuição das limitações funcionais e melhora da qualidade de vida.

\subsubsection{Hidroterapia}

A hidroterapia em gestantes é uma excelente forma para melhorar o condicionamento físico e controlar a frequência cardíaca materna e fetal. Outros efeitos relatados na literatura incluem o aumento da diurese, diminuição de edema e redução da sensação de peso, promovendo relaxamento corporal (BARROS et al. 2018).

O estudo de Sebben et al. (2011), foi feito com duas gestantes, primíparas, que apresentavam dor em região lombar, ocasionadas pelas mudanças estruturais da gravidez e que estavam na 12 e 36 semanas de idade gestacional. A dor foi mensurada pela escala visual analógica, o exame físico constou de teste de flexibilidade do terceiro dedo-solo, avaliação da amplitude de movimento da coluna lombar com goniômetro em todos os movimentos e avaliação postural (vista anterior, posterior e perfil). Após avaliação, iniciou-se o protocolo de hidroterapia, o qual teve duração de dois meses, sendo realizados dez atendimentos com duração de 50 minutos cada, dividido em 3 fases inicial (exercícios de aquecimento), intermediária (exercícios de alongamento e localizados) e final (exercícios de relaxamento). O nível da água foi na altura do peito das gestantes e executadas duas séries de 10 repetição cada. A hidroterapia mostrou-se altamente eficaz na redução do quadro álgico lombar, minimizando os desconfortos musculoesqueléticos ocasionados pela gestação.

Por outro lado, Souza et al. (2012), realizaram um estudo comparativo entre hidroterapia e exercícios realizados no solo. O protocolo foi aplicado em uma gestante, primípara, com 32 semanas de idade gestacional. Inicialmente foi feita avaliação para controle da melhora clínica por meio da eletromiografia dos músculos paravertebrais, perimetria dos membros inferiores (realizada em ortostatismo), para verificar edema, além de mensurar a dor pela escala visual analógica. Por conseguinte, as sessões dos exercícios terapêuticos tiveram duração de 50 minutos e compreendiam 3 fases: aquecimento, alongamento e relaxamento. Iniciou-se com a hidroterapia, a temperatura da água a $34^{\circ}$ graus e altura no processo xifoide. Foi realizado o mesmo protocolo de exercícios na piscina e no solo, com o mesmo número de série, repetição e tempo de intervalo de 15 dias entre um e outro e uma sessão de cada. Os autores chegaram à conclusão que a hidroterapia proporcionou melhores resultados, em relação ao solo, na diminuição do edema gravitacional dos membros inferiores, na tensão dos músculos dorsais e, consequentemente, da intensidade das dores lombares na paciente em questão.

Corroborando com esses achados, Barros et al. (2018) constataram que a hidroterapia foi eficaz no tratamento da lombalgia gestacional e que esta modalidade terapêutica 
pode ser indicada para o alívio das dores lombares e melhora da qualidade de vida da gestante. Este estudo foi realizado com uma gestante de 18 semanas de idade gestacional. Para avaliação foram utilizados os questionários SF-36, McGill, a escala visual analógica e o teste de provocação de dor lombar. Antes de iniciar e ao finalizar o tratamento foi mensurada a pressão arterial, a piscina estava com temperatura entre 27 ${ }^{\circ} \mathrm{C}$ e $33{ }^{\circ} \mathrm{C}$, a duração do atendimento foi de 45 minutos, distribuídos em: 5 minutos de aquecimento; 10 minutos de alongamento; 20 minutos de fortalecimento; 10 minutos de relaxamento e alongamento. $\mathrm{O}$ protocolo foi realizado durante 2 meses, duas vezes por semana, e ao final do mesmo, a paciente foi reavaliada com os mesmos instrumentos.

\subsubsection{Reeducação Postural Global (RPG)}

Um método utilizado para tratar dor lombar na gestação é a RPG. De acordo com seus princípios, as cadeias musculares são constituídas por músculos gravitacionais que trabalham de forma sinérgica dentro da mesma cadeia. Essa técnica preconiza a utilização de posturas específicas para alongamento de longa duração dos músculos (aproximadamente 15 minutos em cada postura). Esse tipo de alongamento pode ser benéfico para gestantes com dor lombar, pois o aumento da lordose lombar é comum nesse período, e a RPG contribui para diminuir e harmonizar as tensões musculares na cadeia posterior, principalmente nos músculos paravertebrais da região lombar. Vale ressaltar que o alongamento global também poderá contribuir para melhor alinhamento corporal, pois durante a gestação o aumento do peso corporal provoca mudança do centro de gravidade, interferindo na postura, equilíbrio e locomoção (GIL et al., 2011).

No estudo de Gil et al. (2011) foi feita uma comparação do grupo controle (GC) com o grupo estudo (GE) de gestantes, com idade gestacional entre 20 e 25 semanas, que possuíam lombalgia. Cada grupo era composto por 17 gestantes. As gestantes do GE fizeram 8 sessões de RPG com duração de 40 minutos, utilizando as posturas fechamento do ângulo coxofemoral, com abdução dos membros superiores e fechamento do ângulo coxo-femoral, com adução dos membros superiores. As gestantes do GC não realizaram nenhum tipo de intervenção. Constatou-se que o GE apresentou diminuição do quadro álgico e das limitações funcionais, já no GC foi observada piora na intensidade da dor e das limitações funcionais.

Resultados semelhantes foram encontrados por Marchiori et al. (2020), que realizaram estudo com uma gestante no terceiro trimestre de gestação, diagnosticada com lombalgia gestacional. A intervenção foi dividida em três etapas: a primeira consistiu na avaliação, dotada de alguns testes especiais e testes de encurtamento muscular: Patrick, Milgran, Tração do Nervo Femoral, Gillet, Valsalva, Laseque, Mobilidade de Quadril, Flexão em Pé, Flexão Sentado, Hoover e Slump. Além disso, foi aplicada a escala visual de dor e o questionário Roland Morris, para avaliar o grau de incapacidade física. A segunda etapa do estudo envolveu 9 sessões de RPG, com duração de 60 minutos cada. Foram utilizadas as posturas "rã no solo" e "em pé no centro", na frequência de duas vezes por semana, com tração cervical e lombar. Por fim, a terceira etapa compreendeu de uma reavaliação com a mesma metodologia inicial. A partir dos resultados, os autores concluíram que a RPG reduziu a dor lombar da gestante estudada, pois a escala de dor passou de dez para zero, todos os testes especiais inicialmente positivos se apresentaram negativos, e o escore do questionário de Roland Morris passou de 16 para 4, refletindo uma diminuição das limitações funcionais.

\subsubsection{Pilates}

Atualmente um dos exercícios mais utilizados na prática clínica é o Pilates, idealizado pelo alemão Joseph Hubertus Pilates. Trata-se de um método de treinamento físico e mental que reorganiza o centro de força como o abdome, quadril e lombar, através da execução de exercícios variados, com poucas repetições, concentração, precisão de movimento, respiração, centralização e fluidez, melhorando a postura e condicionamento físico. Acredita-se que o Pilates pode prevenir ou amenizar as dores na coluna vertebral, estimular a circulação, melhorar a flexibilidade muscular e amplitude de movimento (JUSTINO; PEREIRA, 2016).

Justino e Pereira (2016) realizaram um estudo clínico controlado e randomizado com 15 gestantes entre $13^{\mathrm{a}}$ e $36^{\mathrm{a}}$ semanas de idade gestacional, divididas em 2 grupos: Grupo Pilates, com sete mulheres e Grupo Controle, com oito gestantes, todas com queixa de dor lombar na gestação. Os dois grupos foram submetidos a avaliação por meio da escala visual analógica para mensurar a dor e uma avaliação estabilométrica para obtenção dos valores de oscilação corporal. A seguir foram realizadas 10 sessões de Pilates, duas vezes por semana, com duração de 60 minutos cada. Os atendimentos constituíram-se de exercícios para fortalecimento, alongamento e relaxamento, por meio da utilização de bolas suíças, fitas elásticas, overballs e rolos. Após as 10 sessões, as gestantes foram reavaliadas quanto a dor e oscilação corporal. Concluiu-se que o programa de Pilates solo reduziu a dor lombar em relação ao grupo controle, no entanto 10 atendimentos não foram suficientes para gerar alterações do centro de oscilação corporal.

\section{Conclusão}

Concluiu-se, a partir desta revisão de literatura, que a hidroterapia, RPG e Pilates proporcionaram melhora significativa da dor lombar durante a gestação, havendo diminuição na intensidade da dor. Cabe destacar, entretanto, que são necessários estudos clínicos adicionais, contendo números expressivos de gestantes, descrição minuciosa do protocolo de avaliação e tratamento para analisar criticamente a eficácia desses métodos (hidroterapia, RPG e Pilates) e investigar a duração de seus efeitos em longo prazo, no 
puerpério.

\section{Referências}

BARROS, F.L. et al. Efeito do tratamento hidroterápico na dor lombar e qualidade de vida de gestantes: estudo de caso. Colloquium Vitae, v.10, n.1, p.74-79, 2018. doi: 10.5747/cv.2018

COTA, M.R; METZKER, C.A.B. Efeitos do método Pilates sobre a dor lombar em gestantes: uma revisão sistemática. Rev. Ciênc. Saúde, v4, n.2, p14-19, 2019.

CUNHA, C.M. P. et al. Estudo comparativo da influência da lombalgia nas atividades de vida diária entre primigestas e multíparas. Fisioter. Brasil, v.19, n.2, p.171-176, 2018.

GIL, V.F.B.; OSIS, M.J.D.; FAÚNDES, A. Lombalgia durante a gestação: eficácia do tratamento com Reeducação Postural Global (RPG). Fisioter. Pesq., v.18, n.2, p.164-170, 2011. doi: 10.1590/ S1809-29502011000200011

JUSTINO, B.S; PEREIRA, W.M. Efeito do método Pilates em mulheres gestantes - estudo clínico controlado e randomizado. Publi. Biol., v.22, n.1, p.55-62, 2016. doi: 10.5212

MADEIRA, H.G.R., et al. Incapacidade e fatores associados à lombalgia durante a gravidez. Rev. Bras. Ginecol. Obstetr., v.35, n. 12, p.541-548, 2013.

MARCHIORI, A.N.R, et al. Tratamento fisioterapêutico pela reeducação postural global na lombalgia gestacional: relato de caso. Rev. Ciênc. Saúde, v.5, n.1, p.1-7, 2020.

SANTANA, L.C; PEREIRA, T.L.S. Benefícios da cinesioterapia como tratamento em mulheres com lombalgia gestacional uma revisão da literatura. Rev. Interciência, v.1, n.1, p.39-47, 2018.

SANTOS, D.H, et al. Estudo comparativo da influência da lombalgia nas atividades de vida diária entre primigesta e multíparas. Fisioter. Brasil, v.19, n.2, p.171-176, 2018.

SCHEFFER, A, et al. A hidroterapia como recurso para o alívio da lombalgia em gestantes. Rev. Perspectiva Ciênc. Saúde, v.3, n.2, p.2-11, 2018.

SEBBEN, V. et al. Tratamento hidroterapêutico na dor lombar em gestantes. Perpesctiva, Erechim, v.35, n.129, p.167-175, 2011.

SILVA, H.R., et al. Benefícios da hidroterapia na lombalgia gestacional: revisão da literatura. Rev. Eletr. Trabalhos Acadêmicos, v.2, n.3, p.1-11, 2017.

SOUZA, D.Q., et al. Estudo comparativo entre exercícios realizados no solo e na água aquecida durante a fase gestacional: estudo de caso. Rev. Univap, v.17, n.30, p.13-21, 2012.

TERRA, D.A.T.; LOPES, R.B.; CAETANO, L.F. Benefícios da reeducação postural global na lombalgia gestacional: revisão de literatura. Corpus Scie., v.11, n.2, p.9-16, 2015.

WITTKOPF, P.G. et al. Características biomecânicas dos pés no período gravídico-puerperal: estudo de caso. Rev. Scie. Med., v.25, n.1, 2015. doi: 10.15448/1980-6108.2015.1.19688 